halten, d. h. ein Anlegen oder Bedecken der Konjunlitiva auf der Sklera zu rerhindern. Es soll die Konjunktira bei keiner Augenbewegung im Stande sein, sich über die Skleralwunde \%u rerschieben. Das Auge wird täglich verbunden und mit einer Silberlösung behandelt.

Magnet-Operation und Röntgenbilder. Zum Nachweis von Fremdkörpern im Auge rieht Kibbe (Arch. of Oplith. Jan. 1900) das Röntgenverfahren dem Asmus'schen Sideroskop vor, ebenso dem grossen Magneten, nud zwar dem ersteren wegen seiner besonclers in einer grossen Stalt anhaftenden Inzurerlässigkeit und dem letzteren wegen der nicht zu leugnenden Gefihrlichkeit. Kibbe nimmt 2 I3ilder auf derselben Platte, indem er die Rülıre bei der 2. Aufnahme etwals vou ilurer urspruinglichen Iage verrickt. Als „Finder“ benut\%t er eine grosse Nalel, die in einem Stiick Heftpflaster rertical den geschlossenen Lidern aufliegt; hierdurch ist eine genauere Lokalisation des Fremolkörpers in seinem Verhältnis sowohl zum vertikalen, als auch horizontalen Meridian, als auch in Bezug auf die Tiefe seiner Iage im Auge ermöglicht. Den Vorwurf, dass die Anwendung der Röntgenstralıleu zu kompliziert sei und zu viel Zeit erfordere, weist er durch die Mitteilung eines Falles zurïck. Es nahm ihn genau I Stunde und 10 Minuten in Anspruch, um einen Patienten, der zum ersten Male ihn konsultierte, zu untersuchen, mit dem Röntgenverfahren den Fremdkörper nach\%uweisen und mit dem grossen Magneten zu entfernen. Für die Aufnahme des Rüntgenbildes, die Entwicklung und Fixicrung der Platte rechnet er einen Zeitaufwand ron 20 Ninuten. Den neu eingeführten Wehnelt-Unterbrecher betrachtet er als eine grosse Vereinfachung und „Entteuerung“ des Röntgenrerfahrens.

\title{
IV. \\ Bericht über die englische ophthalmologische Litteratur \\ (II. Semester 1900).
}

\author{
Erstattet von
}

1). GRUBER-London.

Ceber Augenaffection bei Meningitis.

A. Quarry Silcock lat die sehr scltene Complication einer Meningitis mit doppelseitiger Panophthalmitis beobachtet ('Transactions Ophth. Society). In diesem Falle konute im Auge der „Streptococcus lanceolatus" nachgewiesen werden, auf den bereits Uhthoff und $\Delta x$ enfeld in diesem Zusammenhang hingewiesen haben. Nettleship macht auf eine Eigenthümlichkeit des Hypopyous in derartigen Fiallen aufmerksam: dasselhe erscheine weniger eitrig, als geronnenem Eiweiss ähnlich und verschwinde gewöhnlich nach $2-3$ Tagen, wenngleich die Gilaskörpereiterung fortschreite und zum Verlust des Auges führe. 
Interessant ist auch cin ron Sidney Stephenson veröffentlichter Fall (Transactions), bei dem es im Anschluss an Basalmeningitis zur descendirenden optischen Seuritis und diffuser Úveitis kam; im meningealen Exsudat liess sich der Weichselbaum'sche Meningococcus nachweisen, aber nicht im Auge. In diesem /usammenhang sei auch auf die Arbeiten von E. W. Brewerton und namentlich ron Percy Flemming über den Zusammenlıang ron sogenanntem Pseudogliom mit Irningitis hingewiesen. Let\%terer führt mehrere Grüncle an, welche gegen die Erklärung des Pseudoglioms als Product absteigender Entzündung sprechen. lir meint, lass beidle Processe auf gemeinsame septische oder päimische Grundursachen zurïckzuführen seien, und rerweist auf die grosse klinische Aehnlichkeit des meningitischen und septischen Symptomenbildes. Namentlich sei immer an Otitis merlia als Grundursache $\% u$ denken, die auch häufig das Bindeglied zwischen den acuten İanthemen und dem Pseudogliom aljgeben könne. Beachtenswerth sei auch der quoad vitam verhältnissmässig günstige Verlauf des J'seudoglioms bei angeblich primiirer Meningitis, der in den ron F. beobachteten Fällen durch spontane Perforation des Trommelfells auffallend günstig beeinflusst wurde.

\section{Verletzungen des Auges.}

Zum 'Theil im Anschluss an den siidafrikanischen Krieg ist eine reichhaltige Casuistik publicirt worden, aus der nur folgende Beitrüge erwilint seien:

F. Menteith Ogilvie bringt in den Transactions of the Ophth. Soc, einen interessanten, sehr reich und gut illustrirten Artikel über "Lücher" in der Macula im Anschluss an Verletzungen. Jecler hat wohl solche, gerade die Macula betreffenden Flecken schon zu sehen Gelegenheit gehabt, rund oder oral an Gestalt, wie mit dem Locheisen herausgeschlagen, saturirt roth gefürlst und binter dem Niveau der begrenzenden Netzhaut gelegen. Verf. hat 15 einschlägige liälle beobachtet und bringt sie, je ${ }^{\circ}$ ach liehlen oder Vorhaudensein ron deutlicher Netæhautabheloung, in 2 Gruppen. Der Linfang der beobachteten ${ }_{n}$ Löcher" viriirte zwischen 1/2 und eivem gimzen Papillendurchmesser, und die Nireaudifieren\% entsprach in Fiallen ohne deutliche Netzhautabhebung 1-2 Dioptrien. Alle Fïlle hatten centrales Skotom, und die Sehschïrfe war manchmal verhältnissmässig beträchtlich. Verf. betont, dass das Aussehen des Auges ein eigenthïmlich constantes sei; dass die beschriebenen Veriinderungen sich durch das ganze Leben orler wenigstens durch Jahre erhalten; dass die ophthalmoskopischen Verinderungen sofort nach der Verlet\%ung auftreten; dass die Allgemeinstörung des Auges im Inschluss an die Verletzung gering sei und dass ein entsprechendes ophthalmoskopisches Bild nur durch Verletzungen hervorgerufen werde.

Achnliche Verinderungeu beschreibt auch E. Treacher Collins ('Trunsactions).

Exophthalmus und Enophthalmus.

Interessante experimentelle Studien hat Walter Edmunds zu diesem Punkte angestellt. Ausser der Wiederholung der bekannten 
Experimente der Reizung und Durchschneidung des Halssympathicus an Aften, hat Verf. die Versuche Cunningham's mit Verfütterung grosser Mengen Schilddriisenextrakts wiederholt und Proptosis, Mydriasis, Erweiterung der Lidspalte etc. erhalten. I) Resultate nach vollständiger und theilweiser Exstirpation der Schilddrüse waren hingegen bei seinen zahlreichen Experimenten höchst widerspruchsreich.

Lawford hat einen Fall von Exophthalmus nach ThyroidextraktBehandlung eines Myxoedems gesehen. - Geo Mackay hat bei Exophthalmus bösartigen Charakters mit beginnender Hornhautverschwärung nach der Exstirpation der halben Schilddriise ausgezeichneten Erfolg gehabt.

Augenkrankheiten in Aegypten.

Kenneth Scott berichtet in der Lancet in anziehender Weise über seine Erfahrungen während seiner achtjährigen Dienstzeit im "Kasr-el-Aini“-Hospital in Kairo. Erwähnt sei das auffallend häufige Vorkommen höchst maligner aclter Conjunctivitis ohne Gonokokkenbefund, die in nahezu einem Drittel der Fälle zu HornhautVerschwärung führte. Bacteriologische Befunde liegen leider nicht vor. Verf. meint, das Trachom werrle in der Regel durch Berührung mit dem Finger etc., selten durch Fliegen, wie häufig irrthümlich behauptet wurde, übertragen. Uebrigens hatten in seinen Fällen pathologische 7ustände der Lider den Erfolg ron Bulbusoperationen nicht wesentlich beeinflusst. Ein besonderes Kapitel wird der einheimischen Kurpfuscherei gewidmet, namentlich dem Lieblingsmittel der Eingeborenen, dem sogenannten "Sheeshm", das aus Alaun, Ingwer, Cayennepfeffer, manchmal sogar aus ungelöschtem Kalk besteht und das häufige Vorkommen von Symblepharon bei den Eingeborenen leicht verständlich macht. Eine locale Augenaffection ist das „Benga nayra“, tumorartige Randwucherungen, die in acht Fällen operatir entfernt wurden. Erwähnt sei auch das Vorkommen von Perinuclear-Cataract bei jungen I.euten, die au Anaemie in Folge ron ,Bilharzia haematobia" leiden.

\section{Klinische Beobachtungen.}

Erwähnt seien: W. M. Beaumont über Familientendenz zur Ophthalmoplegia externa mit interessantem Stammbaum. E. Treacher Collins zwei Fiille ron cougenitalen symmetrischen Kerben der unteren Lider, nahe dem äusseren Lidwinkel (Transactions). R. Wallace Henry Amblyopie in Folge excessiven Theetrinkens (Ophth. Review). R. W. Do y n e über recuriereude superficielle Hornhautnekrose ('Transactions). Nettleship über das Verschwinden von vorher constatirten markhaltigen Faserflecken - die nicht mit der Papille zusammenhingen - bei entzündlicher Opticusatrophie in zwei Fällen. John Griffith über den Zusammenhang ron Iritis und Gonorrhoe. Kilinisch und anatomisch behandelu an der Hand eines reichen Materials E. Treacher Collins und C. Devereux Marshall die Frage nach der Natur der primären Sehnerventumoren ('Transactions). J. B. Lawford berichtet über 5 Fälle von interstitieller Keratitis bei acfuirirter Syphilis, darunter eine bei einem Arzt, bei dem sich der Primäraffect in der Bindehaut 
entwickelt hatte (im Anschluss an die Operation eines syphilitischen Patienten). J. Work Dodd kommt nochmals auf die Frage nach der klinischen Bedeutung des ,Grünsehens" als Symptom zurück und stellt 13 einschlägige Fälle aus der Litteratur zusammen. Fr meint, dasselbe sei zumeist auf Veränderungen im Sehnerven oder der Netzhaut zurückzufiihren und rechtfertige wahrscheinlich eine ungünstige Prognose ('Iransactions Ophth. Soc.).

\title{
Therapie.
}

Bezüglich der Behandlung der Thränensackleiden hat bei Gelegenheit der Sitzung der ophthalmologischen Section der „British Merlical Association" eine interessante Discussion stattgefunden. G. A. Berry meint, solche Fälle würden in der Regel ïberbehandelt. Meist sei die Ursache nicht Stenose, sondern ein einfacher Katarrh. Dicke Sonden seien überhaupt zu vermeiden. Sondiren nach Schlitzung des oberen Thränenröhrchens solle nicht häufiger als $3-4 \mathrm{mal}$ in Zwischenriiumen ron 14 Tagen vorgenommen werden, und Ausspritzungen seien nur in Fällen ron Ektasie des Thrïneusackes angezeigt. In chronischen Fällen sei wahrscheinlich Exstirpation des Thränensackes das beste. St. Clair Thomson hat niemals floride Affection in der Nase gefunden, meint aber trotzlem, die primäre Infection finde in der Regel ron dort aus statt. Bower gebraucht nur sehr seltep Sonden und iiberlässt acute Fälle, nach Entleerung des Eiters, in der Regel sich selbst. Andere allerdings empfehlen Erweiterung und Einführung dicker Sonden (Lancet, 18. August 1900).

James Hinshelwood empfichlt Holocain als locales Analgeticum bei acutem Glaucom, es unterstiit\%e auch die Wirkung des Eserins (Ophth. Review. Nov.).

A. W. Doyne hat gute Erfolge ron der strahlenden Wärme, namentlich in Fällen von acuter Iritis und Cyclitis gesehen. Er verwendet eine matte Glühlampe von 16 Kerzen Stärke und applicirt dieselbe Anfangs 4-5 Minuten, bei chronischen Fällen bis zu 20 Jinuten. Bei acuten Fällen wird das Auge mit einem Läippchen bedeckt. und der nachträgliche kffect lässt sich durch Massage oder kalte Augen-I)ouche wesentlich verstärken. I ang bat ron dieser Therapie auch bei interstitieller Keratitis im Resorptionsstadium günstige Erfolge gesehen.

V.

Bericht über die belgische ophthalmologische Litteratur im zweiten Semester 1900.

Von

\author{
Dr. ED. PERGENS.
}

Anatomie.

Gallemaerts, Sur la structure du chiısma optique. Bull. acad. r. méd. Belg. série 4. t. XIV. p. 521.

Gallemaerts hat das Chiasma histologisch untersucht bei vier Individuen, denen ein Auge früher enukleirt worden war oder das 